\title{
Imaging and Quantitative Analysis of the Interstitial Space in the Caudate Nucleus in a Rotenone-Induced Rat Model of Parkinson's Disease Using Tracer-based MRI
}

\author{
Deyong Lvi, 2, 3, Jingbo $\mathrm{Li}^{4}$, Hongfu $\mathrm{Li}^{3}$, Yu Fu${ }^{5}$, *, Wei Wang1, 2, * \\ ${ }^{1}$ Department of Radiology, Peking University Third Hospital, Beijing 100191, China; ${ }^{2}$ Beijing Key Laboratory of \\ Magnetic Resonance Imaging Device and Technique, Beijing 100191, China; ${ }^{3}$ Department of Radiology, \\ Dongying People's Hospital of Shandong, Shandong, 257091, China; ${ }^{4}$ Department of Ultrasound, Dongying \\ People's Hospital of Shandong, Shandong, 257091, China; ${ }^{5}$ Department of Neurology, Peking University Third \\ Hospital, Beijing 100191, China
}

[Received April 4, 2016; Revised June 19, 2016; Accepted June 26, 2016]

\begin{abstract}
Parkinson's disease (PD) is characterized by pathological changes within several deep structures of the brain, including the substantia nigra and caudate nucleus. However, changes in interstitial fluid (ISF) flow and the microstructure of the interstitial space (ISS) in the caudate nucleus in PD have not been reported. In this study, we used tracer-based magnetic resonance imaging (MRI) to quantitatively investigate the alterations in ISS and visualize ISF flow in the caudate nucleus in a rotenone-induced rat model of PD treated with and without madopar. In the rotenone-induced rat model, the ISF flow was slowed and the tortuosity of the ISS was significantly decreased. Administration of madopar partially prevented these changes of ISS and ISF. Therefore, our data suggest that tracer-based MRI can be used to monitor the parameters related to ISF flow and ISS microstructure. It is a promising technique to investigate the microstructure and functional changes in the deep brain regions of $\mathbf{P D}$.
\end{abstract}

Key words: Magnetic Resonance, Parkinson's disease, Interstitial Space, Brain, Diffusion

The interstitial space (ISS) within the brain provides an immediate accommodation space for neural cells and contributes to the physiological and functional homeostasis of the brain, where the flow of interstitial fluid (ISF) is important for nutrient supply, waste removal and intercellular communication [1]. Parkinson's disease (PD) is the primary neurodegenerative disease of the basal ganglia and is characterized by neurotransmitter alterations in the caudate nucleus and selective loss of dopaminergic neurons in the substantia nigra [2-4]. Studies have demonstrated microstructural changes in the
ISS of PD [5, 6]. However, changes in ISF flow within the caudate nucleus of PD have not been reported. The caudate nucleus is vulnerable to the effects of PD and is the target region of several promising therapeutic strategies [7,8]. Therefore, investigating the changes in ISS and ISF flow in the caudate nucleus is necessary to comprehensively understand the mechanisms underlying pathogenesis of PD and optimize the efficacy of therapeutic strategies. The width of the ISS ranges from 38-64 nm, which, therefore, is challenging to image in vivo [9]. To our knowledge, the tracer-based MRI

*Correspondence should be addressed to: Drs.Yu Fu and Wei Wang, Peking University Third Hospital, 49 North Garden Road, Beijing 10019, China. Email: lilac fu@sina.com or medimawei@,bjmu.edu.cn

Copyright: () 2017. This is an open-access article distributed under the terms of the Creative Commons Attribution License (CC BY 4), which permits unrestricted use, distribution, and reproduction in any medium, provided the original author and source are credited. 
technique is a unique in vivo method that can measure both the microstructure of ISS and ISF flow in deep brain regions [10, 11]. In tracer-based MRI, the tracer is introduced into the ISS of the target region, and the radiofrequency signal is assessed using MRI. The tracer concentration is calculated by the signal intensity of the images, and the parameters of the microstructure and flow can be calculated according to the diffusion equation. In the present study, we investigated the changes of ISS in the caudate nucleus in a rotenone-induced model of PD, with or without madopar treatment, using tracer-based MRI.

\section{MATERIALS AND METHODS}

\section{Rotenone-induced injury, madopar treatment rat model and behavioral testing}

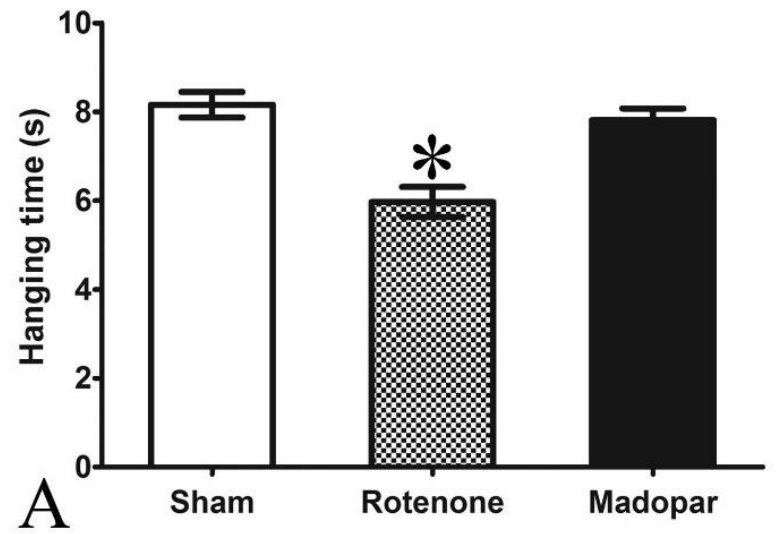

Thirty 8-week-old male Sprague-Dawley rats (280 g-320 g) were randomly divided into three groups $(n=10)$ : (a) rotenone-induced $\mathrm{PD}$ group that received daily subcutaneous injections with rotenone solution (1.5 $\mathrm{mg} / \mathrm{kg} /$ day) [12], (b) madopar-treated group that received both rotenone injections and intragastric administration with madopar $(50 \mathrm{mg} / \mathrm{kg} / \mathrm{day})$ and (c) sham group that received subcutaneous injections with saline. All rats were administrated the above mentioned treatment for 4 weeks. Motor impairment in the rotenone-induced group was verified using the hanging-wire and inclined plane tests $[13,14]$. All animal experimental procedures were reviewed and approved by the Peking University Institutional Animal Care and Use Committee and the Peking University Committee on Animal Care (No. LA2012-016).

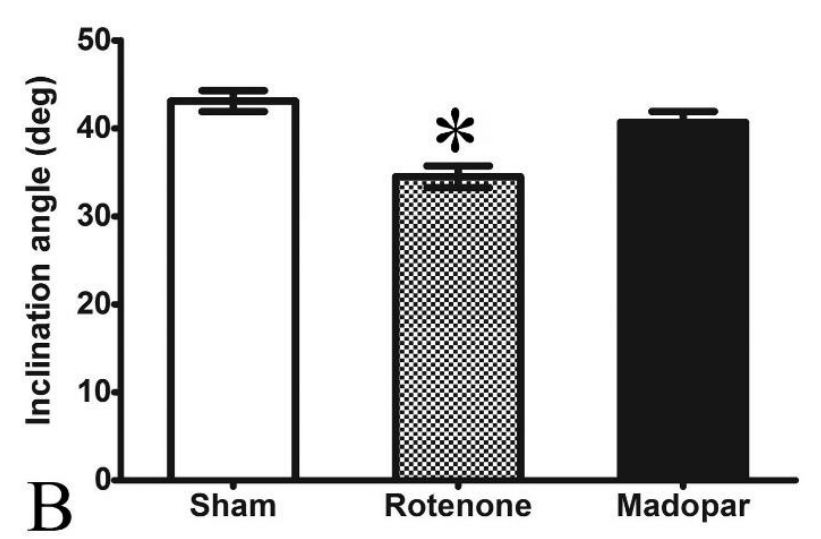

Figure 1. Behavioral changes after treatment. (A) The average hanging time in the hanging-wire test four weeks after rotenoneinduced damage and madopar treatment. (B) The average inclination angle in the inclined plane test four weeks after rotenoneinduced damage and madopar treatment. Both the hanging time and the inclination angle were significantly decreased in the rotenone-induced group compared to the other two groups. Data are the mean \pm SEM $(n=10)$. One-way ANOVA and SNK tests were performed, * represents $P<0.05$.

\section{Tracer-based MRI technique}

The tracer-based MRI technique was perfomed according to the propotocol previously described by Han $[10,15]$. Rats were anesthetized by a combination of pentobarbital sodium, ethanol, chloral hydrate, magnesium sulfate and propylene glycol $(3 \mathrm{ml} / \mathrm{kg})$ via intraperitoneal injection. Anesthesia was subsequently maintained with additional injections over the course of the experiment (approximately $0.7 \mathrm{ml} / \mathrm{kg} / \mathrm{h}$ ). The rats were fixed in a stereotactic apparatus, an incision was made in the scalp along the sagittal suture, then the bregma was exposed. A small trephine hole in the skull bone was made according to the stereotactic coordinates of the caudate nucleus (bregma: $+1.0 \mathrm{~mm}$, lateral: $3.5 \mathrm{~mm}$, vertical: $5.0 \mathrm{~mm}$ ) [16]. $10 \mathrm{mmol} / \mathrm{L}$ of gadolinium-diethylene triamine pentacetic acid (Gd-DTPA) in $2 \mu \mathrm{l}$ was injected into the caudate nucleus of each rat by a syringe pump (rate: 0.2 $\mu \mathrm{L} / \mathrm{min}$ ). After the injection, the needle was left in place for an additional $5 \mathrm{~min}$ and then slowly withdrawn. MR scanning was operated in a 3.0-Tesla MRI system (Magnetom Trio; Siemens Medical Solutions, Germany) with an eight-channel wrist coil using $\mathrm{T}_{1} 3 \mathrm{D}$ MPRAGE sequences. MR scanning was performed sequentially preinjection and post-injection $(0.5,1,1.5,2,3,4,5,6,7$ and $8 \mathrm{~h}$ ). The scanning parameters were as follows: repetition 
time $=1500 \mathrm{~ms}$, echo time $=3.7 \mathrm{~ms}$, flip angle $=12^{\circ}$, inversion time $=900 \mathrm{~ms}$, field of view $=267 \mathrm{~mm}$, voxel $=$ $0.5 \times 0.5 \times 0.5 \mathrm{~mm}^{3}$, matrix $=512 \times 512$ and acquisition time $=290 \mathrm{~s}$. The axial images of the same anatomical site from different time points were imported into the processing workstation for quantitative analysis. The increment in the signal intensity of all of the pixels in the image was recorded after registration and subtraction procession using Matlab (MathWorks, Inc., Natick, MA) and were converted to the concentration of Gd-DTPA [17]. We extracted the parameters related to the microstructure (effective diffusion parameter $D^{*}$ and tortuosity $\lambda$ ) and clearance of Gd-DTPA (clearance rate constant $k^{\prime}$ and half-life $\mathrm{t}_{1 / 2}$ ). $\mathrm{D} *$ represents the diffusion ability of substances in the ISS and is less than the free diffusion parameter (D) due to the diffusion barrier in the ISS. $\lambda$ can be calculated by the equitation $\lambda=\sqrt[2]{\mathrm{D} / \mathrm{D} *}$ and is related to the microstructure of the ISS. $k$ ' refers to nonspecific uptake and represents the loss of Gd-DTPA due to cell metabolism. $\mathrm{t}_{1 / 2}$ is the amount of time needed for a reactant concentration to decrease by half compared to the initial concentration of Gd-DTPA. Almost all GdDTPA is cleared from the ISS through the paravascular spaces surrounding large draining veins and is drained into the cerebrospinal fluid and the lymphatic system [18]. Therefore, $\mathrm{t}_{1 / 2}$ is primarily related to the diffusion and bulk flow of ISF.

\section{Statistical analysis}

One-way analysis of variance (ANOVA) followed by Student-Newman-Keuls (SNK) test was performed for comparisons among the groups with SPSS 18.0. Statistical significance was set a priori at 0.05 .
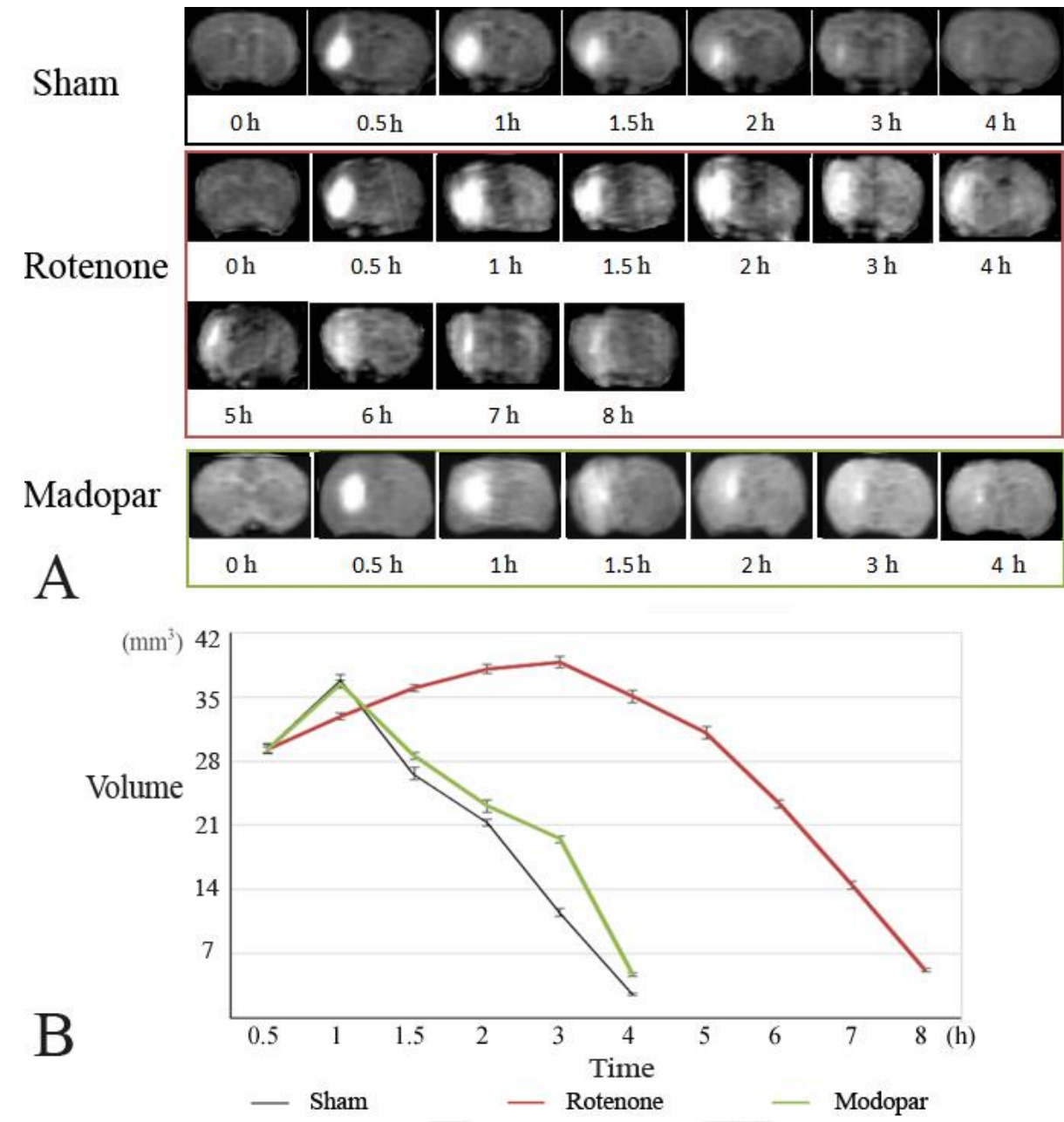

Figure 2. Axial views of MRI and spatiotemporal distribution pattern of GdDTPA after injection into caudate nucleus. (A) Axial MR images of the spreading tracer in the ISS at different time points after the introduction of GdDTPA. Gd-DTPA introduced into the ISS can lighten the water molecules and increase the signal intensity in the spreading regions. The transportation and clearance of Gd-DTPA can be demonstrated using a series of MR images. (B) Line chart of the distribution region at the different time points. The volume of each pixel in the MRI is $0.5 \times 0.5 \times 0.5$ $\mathrm{mm}^{3}$, and the volume amount of the "lightened" regions in each image for each time point was calculated. The maximum spreading region was not significantly different among the three groups. Data are the mean \pm SEM $(n=10)$. SNK test was performed. 


\section{RESULTS}

Compared with the sham group $(8.16 \pm 0.91 \mathrm{~s})$ and the madopar-treated group $(7.81 \pm 0.85 \mathrm{~s})$, the average hanging time in the rotenone-induced model group was significantly decreased $(5.97 \pm 1.07 \mathrm{~s})$ in the hanging-wire test $(P<0.05)$. In addition, compared with the sham group $\left(43.1 \pm 3.8^{\circ}\right)$ and the madopar-treated group $\left(40.7 \pm 3.8^{\circ}\right)$, the average inclination angle in the inclined plane test was significantly decreased in the rotenone-induced group $\left(34.5 \pm 3.9^{\circ}, P<0.05\right)$. There were no significant differences between sham group and the madopar-treated group in either the hanging-wire or the inclined plane test (Fig. 1).

In the sham and madopar-treated groups, a maximum spreading region was reached at 1 and $4 \mathrm{~h}$ after the introduction of Gd-DTPA, and the Gd-DTPA had almost completely disappeared. In the rotenone-induced group, a maximum spreading region was observed $3 \mathrm{~h}$ after the introduction of Gd-DTPA and the tracer was eliminated 8 $\mathrm{h}$ later. The maximum spreading region was not significantly different among the three groups $(P>0.05)$ (Fig. 2)

In this study, we measured parameters related to the microstructure of the ISS $\left(D^{*}, \lambda\right)$ and the clearance of GdDTPA $\left(k^{\prime}, \mathrm{t}_{1 / 2}\right)$. Compared with the sham group, D* was significantly increased $(5.828 \pm 0.727$ vs $2.770 \pm 0.506 \times$ $10^{-4} \mathrm{~mm}^{2} / \mathrm{s}, P<0.05$ ), and $\lambda$ was significantly decreased $(0.916 \pm 0.209$ vs $1.560 \pm 0.320, P<0.05)$ in the rotenoneinduced group. However, compared with the sham group, the amplitude of variation was smaller in the madopartreated group $\left(\mathrm{D}^{*}=3.645 \pm 0.451 \times 10^{-4} \mathrm{~mm}^{2} / \mathrm{s}, \lambda=1.157\right.$ $\pm 0.128, P>0.05)$. Moreover, a significant difference was found between the rotenone-induced group and the madopar-treated group $(P<0.05)$.

The results showed that the $k^{\prime}$ in the rotenone-induced group was significantly decreased $\left(k^{\prime}=0.333 \pm 0.093 \times\right.$ $\left.10^{-4} / \mathrm{s}\right)$, and $\mathrm{t}_{1 / 2}$ was significantly prolonged $\left(\mathrm{t}_{1 / 2}=156.6 \pm\right.$ $10.2 \mathrm{~min})$ in comparison with the sham group $\left(k^{\prime}=0.648\right.$ $\left.\pm 0.082 \times 10^{-4} / \mathrm{s}, \mathrm{t}_{1 / 2}=114.6 \pm 8.7 \mathrm{~min}\right)$ and the madopartreated group $\left(k^{\prime}=0.500 \pm 0.070 \times 10^{-4} / \mathrm{s}, \mathrm{t}_{1} / 2=128.8 \pm 7.1\right.$ min) (Fig. 3).
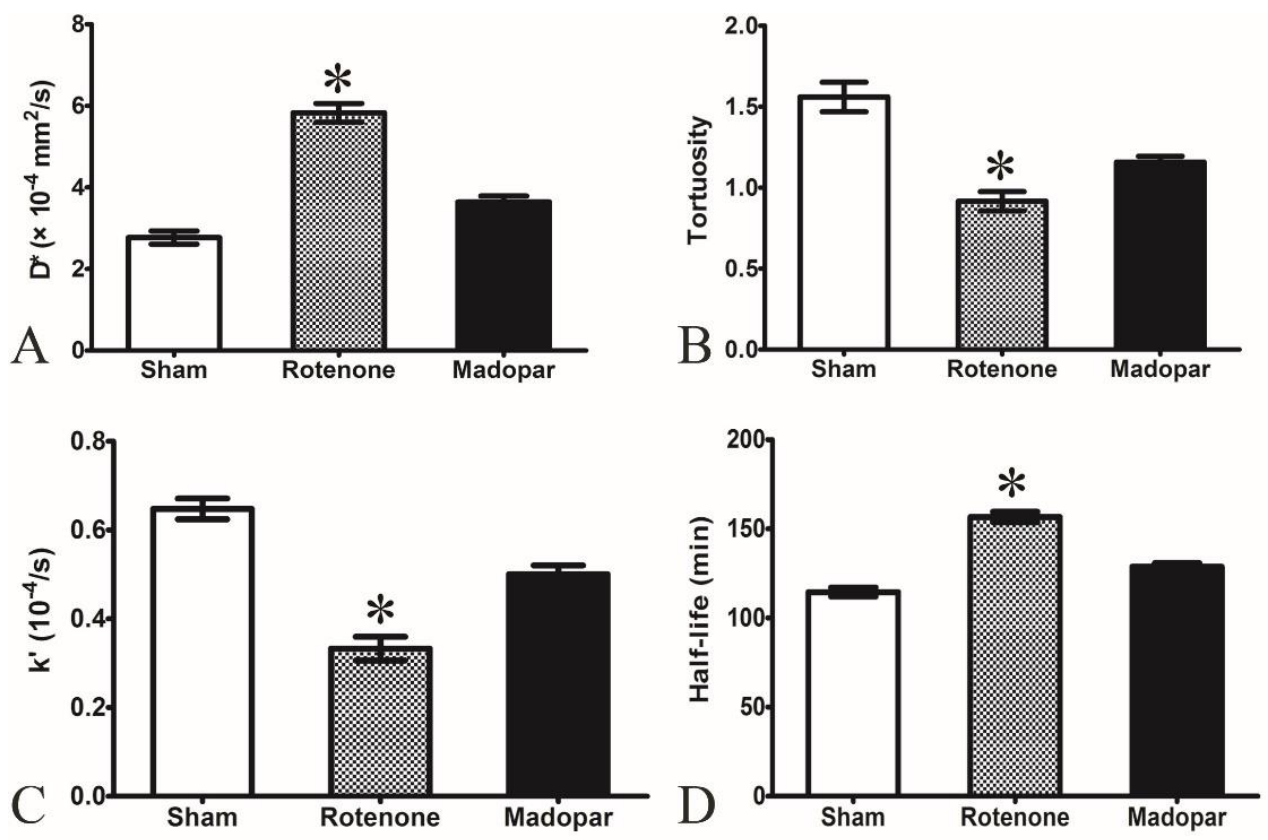

Figure 3. Alterations in the microstructure parameters $\left(D^{*}, \lambda\right)$ and the clearance of Gd-DTPA $\left(k^{\prime}, t_{1}\right)$ in the caudate nucleus after rotenone-induced damage and madopar treatment. (A) Compared to the sham group, the effective diffusion parameter( $\left.\mathrm{D}^{*}\right)$ was significantly increased in the rotenone-induced group. No differences were observed between the sham group and the madopar-treated group. (B) Compared to the sham group, tortuosity $(\lambda)$ was significantly decreased in the rotenone-induced group. No differences were observed between the sham group and the madopar-treated group. (C) Compared to the sham group, the clearance rate constant $\left(k^{\prime}\right)$ was significantly decreased in the rotenone-induced group. No differences were observed between the sham group and the madopar-treated group. (D) Compared to the sham group, half-life $\left(t_{1} / 2\right)$ was significantly prolonged in the rotenone-induced group. No differences were observed between the sham group and the madopar-treated group. Data are the mean \pm SEM $(n=10)$; SNK test was performed. * represents $P<0.05$ 


\section{DISCUSSION}

In this study, using tracer-based MRI allowed us for the first time to visualize the changes in ISF flow in the caudate nucleus in the rotenone-induced rat model of PD. We demonstrated that the ISF flow slowed and that the tortuosity of the ISS significantly decreased in the rat model. Madopar administration partially prevented these changes of ISS and ISF.

Tracer-based MRI was used in the current study, because this technique has the ability to image dynamic ISF flow in the brain from a global view. To date, three techniques have been developed to investigate the ISS in vivo, including real-time iontophoresis (RTI), integrative optical imaging (IOI) and tracer-based MRI. RTI can accurately evaluate the diffusion parameters of the ISS across a distance of approximately 100-200 $\mu \mathrm{m}$, but it cannot visualize ISF flow. Similar to tracer-based MRI, IOI can visualize ISF flow using a fluorescent tracer to calculate the parameters. However, IOI is limited to superficial $(200 \mu \mathrm{m})$ brain regions and cannot be used to investigate the ISS of deep brain regions, where the structure produces too much light scattering [9, 19]. Tracer-based MRI utilizes a magnetic tracer (Gd-DTPA), which can shorten the spin-lattice relaxation time of hydrogen nuclei in water molecules within an effective distance of 2.5 angstroms. This extracellular probe increases the signal intensity of MRI.

According to our findings, the microstructure changes in the ISS measured using tracer-based MRI are consistent with previous reports using RTI [6]. Furthermore, we have verified that there are biophysical changes in ISS in the deep brain nuclei in PD. Importantly, our recent report using a 6-hydroxydopamine-induced rat model of PD demonstrated that there was decreased tortuosity and a reduced clearance rate in the ISS of substantia nigra [5]. The changes were partially mediated by dopaminergic neuron loss and reactive astrogliosis. In the current study, we demonstrated similar changes in the ISS of the caudate nucleus using a rotenone-induced rat model of PD. Whereas, unlike the pathological changes in the substantia nigra, no extensive cell loss or reactive astrogliosis have been observed in the caudate nucleus in PD $[13,20]$. We hypothesize that the changes in the ISS in the caudate nucleus may be caused by the degeneration of dopaminergic axons and the up-regulation of spontaneous neuronal discharge activity $[13,21]$. The boundary structure of the ISS, which is made of the cell membrane and extracellular matrix, prevents the diffusion of substances within the ISS. Moreover, unpublished work from our lab showed that myelin fibers act as a barrier to ISF flow. Axon degeneration can alter brain structures and influence diffusion parameters. Additionally, in a recent study, we demonstrated that functional neuronal discharge activity in normal rats can slow ISF flow and reduce clearance of Gd-DTPA [22].

In future, application of this method will provide new insight into the mechanisms of PD. For example, this technique may be used to answer the following question: "Where PD begins at the cellular level, the neuron soma in the substantia nigra or the axonal terminal in the striatum?". Moreover, current treatment of PD is not clinically satisfied, and pharmaceutical drugs offer only time-limited symptomatic relief and become less effective as the disease progresses [23]. Emerging ISS administrations that involve gene therapies and cell transplantation have shown promise in the treatment of PD [24, 25]. ISS administration provides a number of advantages over conventional administration, including bypass of the blood brain barrier, minor systemic toxicity and enhanced efficacy, etc [26]. Real-time monitoring and quantitative analysis of the changes in the ISS are essential to both optimizing these treatment strategies and evaluating their efficacy. Our results validated the practicality and sensitivity of tracer-based MRI to monitor the changes in the ISS of the deep brain nuclei of treated or untreated PD.

It is important to note that we report only a novel application of tracer-based MRI in this study and future pathological or histological studies require investigations.

\section{Acknowledgements}

We would like to express gratitude to Dr. Han Hongbin for his theoretical guidance, instructive advice and technical support in the design and execution of this study. Dr. Han is the Chief Professor of the Radiology Department of Peking University Third Hospital and the Director of Beijing Key Lab of Magnetic Resonance Imaging Device and Technique. This work was supported by the National Natural Science Foundation of China (No. 81471633 , 91330103,81071148), the Key Project of Peking University Third Hospital (No. BYSY201301), the China Specialized Research Fund for the Doctoral Program of Higher Education (No.20130001130013), and the China Postdoctoral Science Foundation Grant (No. 2015M570901).

\section{References}

[1] Lei Y, Han H, Yuan F, Javeed A, Zhao Y (2016). The brain interstitial system: Anatomy, modeling, in vivo measurement, and applications. Prog Neurobiol, in press.

[2] Labandeira-Garcia JL, Rodriguez-Pallares J, VillarCheda B, Rodriguez-Perez AI, Garrido-Gil P, Guerra MJ (2011). Aging, Angiotensin system and dopaminergic degeneration in the substantia nigra. Aging Dis, 2:257-274 
[3] Rangel-Barajas C, Coronel I, Floran B (2015). Dopamine Receptors and Neurodegeneration. Aging Dis, 6:349-368

[4] Duarte JMN, Schuck PF, Wenk GL, Ferreira GC (2014). Metabolic Disturbances in Diseases with Neurological Involvement. Aging Dis, 5:238-255

[5] Ren RT, Shi CY, Cao J, Sun Y, Zhao X, Guo YF, et al (2016). Neuroprotective Effects of A Standardized Flavonoid Extract of Safflower Against NeurotoxinInduced Cellular and Animal Models of Parkinson's Disease. Sci Rep, 6:22135

[6] Reum T, Olshausen F, Mazel T, Vorisek I, Morgenstern R, Sykova E (2002). Diffusion parameters in the striatum of rats with 6hydroxydopamine-induced lesions and with fetal mesencephalic grafts. J Neurosci Res, 70: 680-693

[7] San Sebastian W, Richardson RM, Kells AP, Lamarre C, Bringas J, Pivirotto P, et al (2012). Safety and tolerability of magnetic resonance imaging-guided convection-enhanced delivery of AAV2-hAADC with a novel delivery platform in nonhuman primate striatum. Hum Gene Ther, 23:210-217

[8] Kells AP, Eberling J, Su X, Pivirotto P, Bringas J, Hadaczek P, et al (2010). Regeneration of the MPTPlesioned dopaminergic system after convectionenhanced delivery of AAV2-GDNF. J Neurosci, 30: 9567-9577

[9] Sykova E, Nicholson C (2008). Diffusion in brain extracellular space. Physiol Rev, 88:1277-1340

[10] Han H, Shi C, Fu Y, Zuo L, Lee K, He Q, et al (2014). A novel MRI tracer-based method for measuring water diffusion in the extracellular space of the rat brain. IEEE J Biomed Health Inform, 18:978-983

[11] Li K, Han H, Zhu K, Lee K, Liu B, Zhou F, et al (2013). Real-time magnetic resonance imaging visualization and quantitative assessment of diffusion in the cerebral extracellular space of C6 gliomabearing rats. Neurosci Lett, 543:84-89

[12] Fathalla AM, Soliman AM, Ali MH, Moustafa AA (2016). Adenosine A2A Receptor Blockade Prevents Rotenone-Induced Motor Impairment in a Rat Model of Parkinsonism. FrontBehav Neurosci, 10:35

[13] von Wrangel C, Schwabe K, John N, Krauss JK, Alam M (2015). The rotenone-induced rat model of Parkinson's disease: behavioral and electrophysiological findings. Behav Brain Res, 279: $52-61$
[14]

Field EF, Whishaw IQ, Pellis SM (2000). Sex differences in catalepsy: evidence for hormonedependent postural mechanisms in haloperidol-treated rats. Behavioural brain research, 109:207-212

[15] Han HB, Li K, Yan JH, Zhu K, Fu Y (2012). An in vivo study with an MRI tracer method reveals the biophysical properties of interstitial fluid in the rat brain. Sci China Life Sci, 55:782-787

[16] Paxinos. G, Watson. C, editors. The Rat Brain in Stereotaxic Coordinates. London: Academic Press; 2007

[17] Liu B, Bai XZ, Zhou FG, Han HB, Hou C (2013). Mutual information based three-dimensional registration of rat brain magnetic resonance imaging time-series. Comput Electr Eng, 39:1473-1484

[18] Iliff JJ, Wang M, Liao Y, Plogg BA, Peng W, Gundersen GA, et al (2012). A paravascular pathway facilitates CSF flow through the brain parenchyma and the clearance of interstitial solutes, including amyloid beta. Sci Transl Med, 4: 147ra111

[19] Xiao FR, Nicholson C, Hrabe J, Hrabetova S (2008). Diffusion of flexible random-coil dextran polymers measured in anisotropic brain extracellular space by integrative optical Imaging. Biophys J, 95:1382-1392

[20] Gates MA, Laywell ED, Fillmore H, Steindler DA (1996). Astrocytes and extracellular matrix following intracerebral transplantation of embryonic ventral mesencephalon or lateral ganglionic eminence. Neuroscience, 74:579-597

[21] Burke RE, O'Malley K (2013). Axon degeneration in Parkinson's disease. Exp Neuro, 246: 72-83

[22] Shi C, Lei Y, Han H, Zuo L, Yan J, He Q, et al (2015). Transportation in the Interstitial Space of the Brain Can Be Regulated by Neuronal Excitation. Sci Rep, 5: 17673

[23] Davie CA (2008). A review of Parkinson's disease. $\mathrm{Br}$ Med Bull, 86:109-127

[24] Chen ZG (2015). Cell Therapy for Parkinson's Disease: New Hope from Reprogramming Technologies. Aging Dis, 6:499-503

[25] Stoessl AJ (2014). Gene therapy for Parkinson's disease: a step closer? Lancet, 383: 1107-1109

[26] Xu F, Hongbin H, Yan J, Chen H, He Q, Xu W, et al (2011). Greatly improved neuroprotective efficiency of citicoline by stereotactic delivery in treatment of ischemic injury. Drug Deliv, 18:461-467 